\title{
Does Panel Type Matter for LCD Monitors? A Study Examining the Effects of S-IPS, S-PVA, and TN Panels in Video Gaming and Movie Viewing
}

\author{
Ki Joon $\mathrm{Kim}^{1}$ and S. Shyam Sundar ${ }^{1,2}$ \\ ${ }^{1}$ Department of Interaction Science, Sungkyunkwan University, Seoul, Korea 110-745 \\ veritatedskku. edu \\ ${ }^{2}$ Media Effects Research Laboratory, College of Communications, \\ Pennsylvania State University, University Park, PA 16802 \\ sss12@psu .edu
}

\begin{abstract}
As computer-based devices become the primary media via which users view movies and play interactive games, display technologies (e.g., LCD monitors) have focused increasingly on quality of video fidelity, with much debate surrounding the relative efficacy of different panel types of LCD monitors. A 3 (S-IPS panel vs. S-PVA panel vs. TN panel) x 2 (game vs. movie) between-subjects experiment was conducted to examine the effects of LCD panel type in facilitating regular viewing as well as enhanced interactive TV experiences. Data from the experiment showed that LCD panel and stimulus type as well as computer literacy were important factors affecting users' viewing and interaction experiences. Limitations and implications for theory and ongoing research are discussed.
\end{abstract}

Keywords: LCD panel, response rate, contrast ratio, viewing angle, computer literacy.

\section{Introduction}

Choosing an LCD monitor that best fits a user's needs and preferences has become relatively difficult in recent years; a number of factors such as screen-size, brand reputation, price, and technical specifications (e.g., response rate, contrast ratio, and viewing angle) are offered as considerations when purchasing a monitor. Although screen-size has been perhaps the most dominant factor affecting consumer's choice since the debut of LCD monitors, recent marketing emphasis on LCD panel types such as S-IPS, S-PVA, and TN has raised the question whether users should factor panel type into their decision-making. Different panel technologies are used in the production of LCD monitors, and manufacturers acknowledge that overall display quality that viewers experience is largely affected by the type of LCD panel to which they are exposed. In other words, LCD panel type is a key factor, according to manufacturers, that determines users' interactive and immersive viewing experiences. Therefore, it should be considered as integral as screen-size and brand reputation. 
However, no empirical study has been conducted to examine the effects of LCD panel type on users' viewing and interacting experiences. As a result, it is unclear whether a particular panel is more effective in providing greater satisfaction, presence, and enjoyment while interacting with LCD monitors. The present study is a modest first attempt at investigating the psychology of LCD monitor users when exposed to three major panel types: S-IPS, S-PVA, and TN.

\subsection{Panel Difference - From an Engineering Perspective}

TN (twisted nematic) panel has been most widely used due to the advantages of its high transmittance, simple fabrication process, and relatively low production cost [1]. The panel's affordable price and fast response rate have made it the most suitable panel for typical office use and fast-paced gaming. The biggest downside of the TN panel, however, is its severe off-axis image deterioration resulting in the worst viewing angle, color reproduction, and contrast ratio in LCD panel technology [2]. The panel is not recommended for movie viewing because, unlike 8-bit S-PVA and SIPS panels that are fully capable of displaying 16.7 million colors in 24-bit true color, it only mimics the 16.7 million colors in 6-bit.

To solve the drawbacks of TN panel, IPS (in-plane switching) panel was first developed by Hitachi in 1996 and later enhanced by LG Display with S-IPS (super inplane switching) technology. The basic principle of the IPS panel was "to change the physical behavior of the liquid crystal layer by having the molecules move in parallel to the TFT and color filter layers rather than at oblique angles," which resulted in "significantly lessened light scattering, and thus improved the picture uniformity and color fidelity when viewed from wide angles" [3]. LG Display further developed the original IPS technology into a premium LCD panel with improved viewing angle, color fidelity, response time, and contrast ratio called S-IPS.

Developed and manufactured by Samsung Electronics as an improved alternative to the existing PVA technology, S-PVA (super patterned vertical alignment) is a new display technology providing image quality advantages over S-IPS, including high transmittance, 2300:1 contrast ratio, and wide viewing angle with no off-axis image inversion [2]. Samsung's new technology called "Magic Speed" claims to offer enhanced response time, rendering the S-PVA panel more suitable for gaming and other interactive applications than the traditional PVA panel.

\subsection{Panel Difference - From a Psychological Perspective}

Previous studies on the relationship between perceived viewing quality (e.g., attention, enjoyment, memory, and presence) and display screen have provided empirical explanations for consumers' preference for large screens and highresolution display devices. Reeves, Detenber, and Steuer [4] showed participants short clips from action films on 35-inch and 70-inch screens, and found that the larger screen elicited a greater level of attention, sense of reality, and presence. Similar studies conducted by Lombard [5] and Detenber and Reeves [6] also found that participants experienced greater enjoyment and arousal and remembered content better when exposed to larger screens. 
High-resolution display is another key factor contributing to greater presence. Bocker and Muhlbach found that participants exposed to higher resolution display in a video conferencing system elicited greater sense of communicative presence [7]. A later study conducted by Neuman also found that high resolution images evoked a higher level of self-reported presence than standard resolution [7].

In accordance with these previous studies that explored the effects of display characteristics on viewers' psychology, the goal of the present study is to identify the effects of LCD panel type, another display characteristic that had never been studied before, on viewers' perceived viewing quality. Therefore, we examine the following research question:

RQ: For LCD monitor users, controlling for content and time spent on the monitor, what is the effect of LCD panel type and stimulus type (Independent Variables) upon viewers' satisfaction with image quality and technical features (i.e., response rate, viewing angle, and contrast ratio) as well as perceived level of presence and enjoyment (Dependent Variables) of movies and games?

\section{Method}

\subsection{Design and Participants}

A fully-crossed 3 (panel type: S-IPS vs. S-PVA vs. TN) x 2 (stimulus type: game vs. movie) between-subjects experiment was conducted to answer the research question. Data were analyzed from 60 undergraduate and graduate students from a four-year university in Seoul who signed up for the experiment through an online registration page posted on the university's main homepage. Exactly 30 men and 30 women (average age of 23) signed up for the experiment. All participants signed an informed consent form prior to their participation.

\subsection{Apparatus}

A 26-inch LG S-IPS panel monitor, a 27-inch Samsung S-PVA panel monitor, and a 26-inch Samsung TN panel monitor were connected to three high-performance desktop computers with identical hardware specifications (i.e., manufacturer, CPU speed, RAM, and graphic card) via DVI connection. Each computer was equipped with a Logitech 5.1-channel surrounding sound headphone. The monitors' brand logos were masked in order to avoid potential effects of manufactures' brand reputation, and the monitors' user-changeable settings (e.g., color tone, brightness, and contrast) were set to the factory standard. Participants in one condition could not see the monitors used in the other two conditions. Above configurations remained the same throughout the experiment.

\subsection{Stimulus Material}

A pursuit scene in downtown Paris from G.I. Joe: The Rise of Cobra was selected for the movie-watching condition. The present study intentionally chose a speedy pursuit scene from the movie, instead of a slow, long-take scene, in order to allow participants to identify potential motion blurs caused by difference in response rate of each monitor. The film was played in 1080p high-definition Blu-ray quality. 
The game used for the gaming condition was Burnout Paradise. This racing game was selected because it was easy to navigate the vehicle using a keyboard and its content was similar to the Paris pursuit scene from G.I. Joe. Burnout Paradise allows players to change the point of view (first or third person) and to select a vehicle that the player wishes to drive. The present study instructed participants to play the game in the first-person point of view based on previous game studies suggesting that playing a game in the first-person point of view resulted in greater involvement and immersion [8].

\subsection{Procedure}

Participants were randomly assigned to one of the six conditions. In the gaming condition, brief instructions about game controls and navigation were provided, along with an opportunity to test drive for one minute in order to build familiarity with keyboard-based controls. Participants were instructed not to change the vehicle and the first-person point-of-view mode while playing the game, but told that they could freely adjust the volume and sitting posture to their comfort level. Once the experimenter finished giving the instructions, participants were told to start playing the game for 10 minutes. The experimenter left the room. After 10 minutes, the experimenter re-entered the room and administered a paper-and-pencil questionnaire measuring participants' level of satisfaction with the monitor they used as well as their perceived viewing/rendering quality.

In the movie-watching condition, participants were told that they were going to watch a scene from G.I. Joe for 10 minutes alone in the room, and instructed not to modify any configurations on the computer while the movie was being played, except the volume and their sitting posture. The experimenter started the movie and then left the room for 10 minutes. The paper-and-pencil questionnaire was administered when the experimenter re-entered the room.

After filling out the questionnaire, participants in both conditions were debriefed, paid five dollars, and asked not to discuss the experiment with others.

\subsection{User Experience (UX)}

The present study created a questionnaire composed of 19 items measuring participants' level of computer literacy and satisfaction with the monitors, as well as perceived level of presence and enjoyment. Participants responded to each item by marking on a 10-point Likert scale (1="strongly disagree," 10="strongly agree").

\section{Results}

A series of General Linear Model analyses was conducted with the two manipulated independent variables (panel type and stimulus type) and one measured variable (computer literacy level) on each of the 19 UX variables.

The analysis found interactions between stimulus type and computer literacy for perceived realism (measured by "During the game/movie, I felt I was in the world the game/movie created"; $\mathrm{F}(1,48)=7.65, \mathrm{p}<.05)$, satisfaction with overall viewing experience (measured by "I was satisfied with the overall viewing experience provided by the LCD monitor"; $\mathrm{F}(1,48)=4.54$, $\mathrm{p}<.05$ ), and presence (measured by 
"When the movie/game ended, I felt like I came back to the real world after a journey"; $\mathrm{F}(1,48)=4.62, \mathrm{p}<.05)$. For participants in the gaming condition, computer literacy level was positively related to perceived realism, satisfaction with overall viewing experience, and level of presence regardless of panel type used for playing the game. For participants in the movie condition, however, a reverse relationship was found, such that the computer literacy was negatively related to these three UX outcomes.

The analysis revealed a three-way interaction predicting users' satisfaction with contrast ratio (measured by "I was satisfied with the contrast ratio of the LCD monitor"; $F(2,48)=4.12, \mathrm{p}<.05)$. In the gaming condition, the relationship between computer literacy and satisfaction with contrast ratio was dependent on panel type, such that computer literacy was positively related to satisfaction with contrast ratio for those using the S-IPS and TN panel, and negatively related to those using the SPVA panel (Fig. 1). For those in the movie-watching condition, computer literacy was negatively related to satisfaction with contrast ratio for those using the S-IPS panel, whereas it was unrelated to those watching with the TN and S-PVA panel.
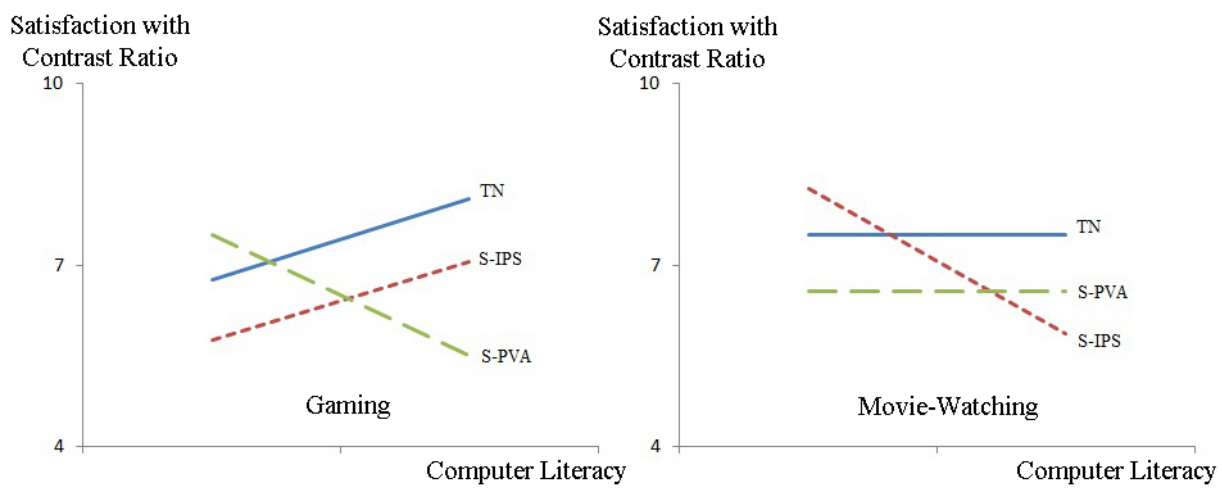

Fig. 1. Three-way interaction for users' satisfaction with contrast ratio

Another three-way interaction was discovered in predicting users' satisfaction with overall viewing experience (measured by "I was satisfied with the visual display quality of the LCD monitor"; $F(2,48)=3.30$, $\mathrm{p}<.05$ ). For participants in the moviewatching condition, computer literacy was negatively related to overall viewing experience whereas it was positively related for those in the gaming condition. This pattern was found only when using the S-IPS panel monitor. There was no such genre-based distinction in the relationship between computer literacy and viewing experience for those using the other two panels.

A three-way interaction for presence (measured by "The game/movie-generated world seemed to me somewhere I really visited") was also significant, $F(2,48)=4.32$, $\mathrm{p}<.05$. In the gaming condition, computer literacy was positively related to presence for those using the S-IPS and S-PVA panel (Fig. 2). For participants in the moviewatching condition, however, computer literacy was positively related to presence for those using the S-IPS panel and negatively for those using the S-PVA panel, whereas it was unrelated to those using the TN panel in both conditions. 

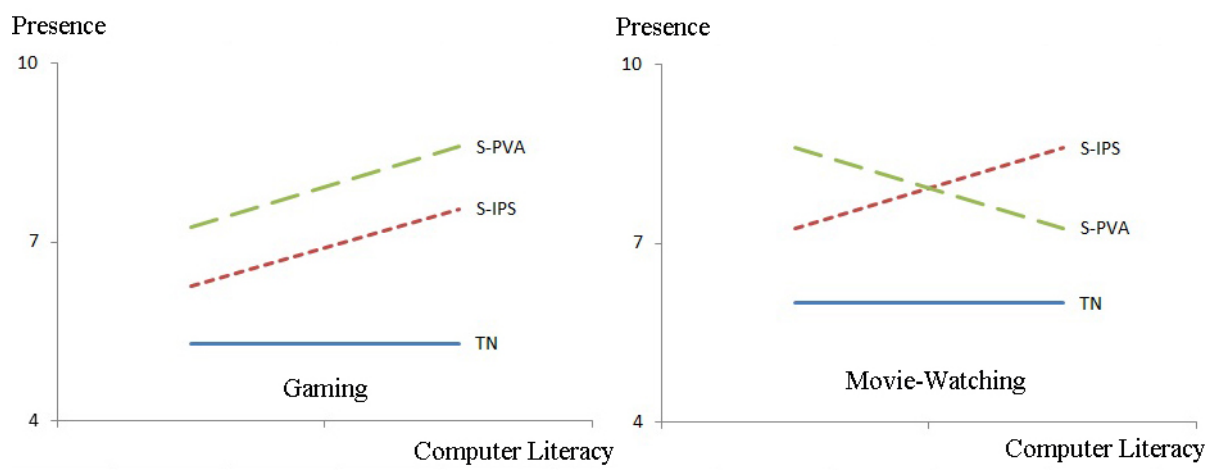

Fig. 2. Three-way interaction for users' perceived level of presence

For enjoyment, a main effect for panel type was found, $F(2,48)=3.47, \mathrm{p}<.05$, such that participants exposed to the S-PVA panel $(M=8.76, S E=.37)$ scored significantly higher on the "I enjoyed playing/watching the game/movie" item than those exposed to the S-IPS (M=7.50, $\mathrm{SE}=.39)$ and TN panel $(\mathrm{M}=7.58, \mathrm{SE}=.39)$. Another main effect revealed that participants in the movie-watching condition $(\mathrm{M}=8.47, \mathrm{SE}=.31)$ scored higher on the item than those in the gaming condition $(\mathrm{M}=7.43, \mathrm{SE}=.32)$, $\mathrm{F}(1,48)=5.38, \mathrm{p}<.05$. Yet another main effect showed that the higher the computer literacy level, the greater the enjoyment, $\mathrm{F}(1,48)=14.38, \mathrm{p}<.001$.

In summary, the present study found that computer literacy is a powerful factor (perhaps even more important than panel type) affecting users' perceived level of realism and presence. It also has effects on monitor users' enjoyment and satisfaction with contrast ratio, a key technical feature of LCD monitors. In general, computer literacy was positively associated with viewing experience, presence and satisfaction with contrast ratio when the IPS panel-type monitor is used in an interactive mode, i.e., for playing games. It is however negatively associated with viewing experience and satisfaction when used in a non-interactive mode, i.e., watching movies. As for the S-PVA panel type, computer literacy is positively associated with presence when playing games but negatively associated with contrast-ratio satisfaction.

\section{Discussion}

Although the present study did not find a particular LCD panel that elicited the highest level of satisfaction, enjoyment, and presence under all conditions, our findings suggest important guidelines and insights for future research on psychological effects of display devices. In general, participants in the present study showed a slight preference for the S-PVA panel over the S-IPS or TN panel types in terms of enjoyment; participants who interacted on a monitor with the S-PVA panel perceived higher level of enjoyment than their counterparts in the other two conditions. However, no main effect for panel type was found on other UX measures. This is because the effects of panel type are qualified by users' prior experience with computing technology. 
This finding encourages consumers to ask a practical question: Is it worth the money? Monitors with PVA panel are generally more expensive than IPS and TN panel monitors. The PVA panel monitor used in the experiment $(\mathrm{MSRP}=\$ 1,100)$ was nearly twice as expensive as the S-IPS (MSRP=\$640) and TN panel monitors $(\mathrm{MSRP}=\$ 480)$. Whether consumers are willing to pay that much more to buy PVA panel monitors for "supposedly" greater enjoyment is questionable because consumers' preference is largely influenced by a combination of product price and visible differences noticed during in-store demonstrations (which may not always be fair comparisons). Given our findings, only high-end gamers who are highly computer-literate may find the presence afforded by S-PVA to be worth the added expense. But then, our interaction findings indicate that IPS panel, which is half the price, will do just as well for gaming (and presumably other interactive TV experiences) as long as the user is computer-literate.

Unexpectedly, computer literacy turned out to be quite a significant predictor. For gaming, participants with higher computer literacy level were more satisfied with their overall viewing experience while experiencing greater realism and presence and were more satisfied with their overall viewing experience. However, a reverse relationship was found for movie-watching. We may interpret this as follows: individuals with high computer literacy level tend to focus more on active interaction with technology (i.e., playing the game using both mouse and keyboard simultaneously), whereas those with low computer literacy level focus more on the content (i.e., movie) delivered to them. This provides valuable insights for future studies on the relationship between computer literacy and technology acceptance.

The implications of the present study suggest that display engineers and manufacturers should be looking more carefully at how panel characteristics (e.g., response rate, viewing angle, and contrast ratio) affect and interact with users' viewing/interaction experience and overall enjoyment. The main effect for stimulus type suggests that interactive use of TV display systems, such as in games, leads to inherently lower ratings of panel characteristics and reported enjoyment. This means designers of display technologies and related systems that enable interactive TV ought to work harder to satisfy users regarding the fidelity of video rendering on their screen. Newer display technologies such as LED (light-emitting diode) and AMOLED (active-matrix organic light-emitting diode) provide avenues for further research. Researchers from psychology and communication may also examine how individual differences such as computer literacy, educational attainment and age affect user acceptance of display technologies that offer slightly superior quality at a heavy price.

A notable limitation is that the present study allowed first-person point of view in the gaming condition. While playing the game in first-person is likely to result in greater involvement and immersion in the game [8], the movie was shot and played with the third-person point of view, thus producing a confound. A recent study on game and violence suggested however that playing a game in third person, not in first person, resulted in increased involvement and focus [9], which might explain why participants enjoyed watching the movie significantly more than playing the game.

Ongoing research will further probe the impact of panel type on viewing-angle satisfaction and content enjoyment, as well as the relationship between these dependent measures. We will also take into account the size of participants' own 
computer monitors and whether they are aware of LCD panel differences, and analyze these variables as covariates in the relationship between the panel type and viewing experience.

Acknowledgments. This study was supported by a grant from the World-Class University program (R31-2008-000-10062-0) of the Korean Ministry of Education, Science and Technology via the National Research Foundation. The authors wish to thank Sungyeon Kim and Sanghun Kwak, Ph.D. students at the Department of Science, Sungkyunkwan University, for their collaboration in the data collection process.

\section{References}

1. Yoon, S., Won, T.: Electrode Structure for High Transmittance and Aperture Ratio in TFTLCD. Journal of Materials Processing Technology 191, 302-305 (2007)

2. Lyu, J., Sohn, J., Kim, H., Lee, S.: Recent Trends on Patterned Vertical Alignment (PVA) and Fringe-field Switching (FFS) Liquid Crystal Displays for Liquid Crystal Television Applications. Journal of Display Technology 3, 404-412 (2007)

3. Cleverdis Special Report, http: / /www.cleverdispdfdownloads.com/pdf_files/ spr_lpl_05.pdf

4. Reeves, B., Detenber, B., Steuer, J.: New Televisions: The Effects of Big Pictures and Big Sound on Viewer Responses to the Screen. In: 43rd Annual Conference of the International Communication Association, Washington, D.C (1993)

5. Lombard, M.: Direct Responses to People on the Screen: Television and Personal Space. Communication Research 22, 288-324 (1995)

6. Detenber, B., Reeves, B.: A Bio-informational Theory of Emotion: Motion and Image Size Effects on Viewers. Journal of Communication 46, 66-84 (1996)

7. Lombard, M., Dittion, T.: At the Heart of It All: The Concept of Presence. Journal of Computer Mediated Communication 3 (1997)

8. Tamborini, R., Eastin, M., Lachlan, K., Skalski, P., Fediuk, T., Brady, R.: Hostile Thoughts, Presence and Violent Virtual Video Games. In: 51st Annual Conference of the International Communication Association, Washington, D.C (2001)

9. Farrar, K., Krcmar, M., Nowak, K.: Contextual Features of Violent Video Games, Mental Models, and Aggression. Journal of Communication 56, 387-405 (2006) 